ORIGINAL ARTICLE

\title{
Partiality of the General Population towards Abortion and the Awareness Regarding it: A Cross Sectional Study
}

\author{
MINAHIL AFTAB ${ }^{1}$, ROBASS ZIA ${ }^{2}$, KURRAT UL AAIEN ${ }^{3}$, REHAN ZAFAR ${ }^{4}$, QUDSIA UMAIRA KHAN ${ }^{5}$ \\ $1,2,3,42^{\text {nd }}$ year MBBS, CMH Lahore Medical College and Institute of Dentistry, Lahore, Pakistan. \\ ${ }^{5}$ Assistant Professor, Department of Physiology, CMH Lahore Medical college and Institute of Dentistry, Lahore, Pakistan. \\ Correspondence to Dr. Qudsia Umaira Khan, Email: drqudsia@yahoo.com, Cell: 0300-4816979
}

\begin{abstract}
Background: Abortion is the termination of a pregnancy by expulsion of an embryo or foetus. In Pakistan, $54 \%$ of the 4.2 million unintentional pregnancies were terminated deliberately. Given the taboo around the subject, abortions are filled with unnecessary risk and fear, legal and physical. Our work intends to shed light on the factors that limit this taboo, exploring the line beyond which abortion is considered acceptable.

Aim: To explore the limits of the taboo around abortion; to explore the extent to which certain taboos apply and deduce circumstances that deem abortion an acceptable practice.

Methods: A sociological and statistical snow-ball type of semi-quantitative, semi-qualitative study was conducted. The study was carried out on the general adult Pakistani population, with focused groups of medical students, non-medical students, and non-students belonging to different employment status. A questionnaire-based survey was implemented. Several people were interviewed as well.

Conclusion: The majority of participants were accepting of abortion in case of severe physical health issues. For questions involving mental health, majority of the population disagreed with abortion as a considerable option. However, the entire population strongly agreed with the fact that awareness regarding abortion is inadequate and there needs further light to be shed on it.
\end{abstract}

Keywords: abortion, taboo, mental health, awareness

\section{INTRODUCTION}

Abortion is a term difficult to precisely define ${ }^{1}$. The general scientific understanding of abortion relates to the ending of the progression of fetal development, prior to birth and delivery, to the effect that a live birth is not possible. Medically, it is the deliberate termination of a human pregnancy, most often performed during the first 28 weeks of pregnancy ${ }^{2}$.

Roughly 121 million unintended pregnancies occurred each year between 2015 and 2017. Out of which 46 million were intentionally terminated each year. Among these, $56 \%$ had been conducted legally while $44 \%$ illegally. Likewise, majority of these abortions took place in developing countries with the highest rate being in eastern Asia and the lowest in Oceania ${ }^{3}$.

As of Pakistan, in 2012, there were approximately nine million pregnancies, of which 4.2 million were unintended. Of these unintended pregnancies, $54 \%$ resulted in induced abortions and $34 \%$ in unplanned births ${ }^{4}$.

There are various methods for induced abortions depending on the individual's own health and stage of pregnancy during the procedure: a) the medical abortion is performed by taking mifepreston (Mifeprex) and misoprostal (Cytotec) orally within the first 10 weeks of gestation. In b) Methotrexate and misoprostol (MTX) method, the two drugs aforementioned are taken orally within the first seven weeks of pregnancy usually in the case of ectopic pregnancy (EP). c) Vacuum aspiration is a method commonly used to terminate pregnancy within the first trimester or early second trimester. The procedure involves a suction pump that gently sucks the foetus and

Received on 13-04-2021

Accepted on 23-08-2021 the placenta out of the uterus through the vagina. The procedure lasts for up to 5 to 10 minutes. d) the Dilation and evacuation method of abortion is practiced in women who have delayed their pregnancy or wants to end it because the foetus has severe abnormalities. It is conducted in the second trimester usually after the 14th Week ${ }^{5,6}$. The procedure involves a combination of vacuum aspiration, forceps and cervical dilation, lasting for up to 30 minutes. e) the last method for planned abortion is the induction method which is considered as the last ditch stand to conduct an abortion after 24th week of pregnancy. Labour is induced by oral pills and the foetus is taken out bit by bit using a suction pump or a curette. The procedure may take several hours or even a day ${ }^{7}$.

In addition to being a nuisance to define, it also carries with it significant cultural baggage. People's socioeconomic status, their religion and their political affiliations all play a crucial role in determining their attitudes towards abortion. These attitudes can also vary depending on the rationale behind the abortion. In general, abortions that are medically warranted tend to treated better than those that are freewilled. However, even within the set of abortions that are medically warranted and advisable, there exists a hierarchy ${ }^{8}$. Abortions that are necessitated by physical process - injurious to the mother or the foetus - are accepted warmly. Alternatively, abortions that are the result of mental or psychological incapacitations or disabilities are widely considered illegitimate ${ }^{9}$.

Our work intends to elaborate and explore this phenomenon. Why are physical reasons for abortion easier to justify as opposed to mental, even when the impacts on the mother and child would be the same? Are some psychological reasons that are easier to justify than others? Through a series of terse and exacting questions, in a simple survey-based format, we tried to peel back the layers to come to a conclusion as to what factors can 
render an abortion valid, and others reprehensible. Furthermore, we amassed public's disposition to spread awareness regarding the legitimacy of abortion.

\section{MATERIAL AND METHODS}

A sociological and statistical snow-ball type of semiquantitative, semi-qualitative study was conducted on students of multiple medical and non-medical undergraduate institutes and among adults of varying employment statuses, with a calculated sample size of 343 individuals according to the WHO formula. The study was conducted in $\mathrm{CMH}$ Lahore Medical College and Institute of Dentistry, Lahore after permission from Ethical Review Board with a study duration of 3 months starting from 18th September, 2020 till 20th November, 2020. A formal standardized (quantitative) questionnaire; comprising scaled questions was assembled based on which a survey was held and the data was further analysed using Microsoft Excel version 2019 and SPSS version 23. In order to evaluate the discernibility level and to eliminate any kind of partiality in the nature of the questionnaire, we personally interviewed various people and on the basis of their views about the questions, we made certain changes in the questions to make the questionnaire more digestible and unbiased. Furthermore, we translated all the questions in Urdu as well to dispel any kind ambiguity in the minds of the individuals taking part in the survey.

Inclusion criteria were 1) sex (male/ female/ others), 2) age above 18 years, 3) type of education (medical sciences/ mathematics major/ management sciences), 4) employment status (employed/ unemployed/ selfemployed), 5) monthly family income (within any of the given ranges) whereas the exclusion criteria was 1) age less than 18 years.

The research posed no risk to the participants and the study was conducted with their consent. Moreover, the research did not involve the vulnerable population including children, prisoners, handicapped or mentally disabled persons, or pregnant women hence an IRB approval is not required.

\section{RESULTS}

The study was conducted on multiple medical and nonmedical undergraduate institutes and among adults of varying employment statuses, with a calculated sample size of 343 individuals according to the WHO formula. The participants belonged to both genders, out of which $60.2 \%$ were female and $39.8 \%$ were male (Figure 1). Among them $73.9 \%$ were students and $24.4 \%$ were not (Figure 2a). Furthermore, among the students, $55.2 \%$ were studying medical sciences while $44.8 \%$ had either mathematics or management sciences as their core subjects (Figure 2a (i)). Meanwhile, among the non-student population, $34.9 \%$ people were employed, 54\% were unemployed and $11.1 \%$ were self-employed (Figure 2a (ii)).

In response to monthly household income in $P K R$, $35.8 \%$ had income below $1,00,000,33.8 \%$ had between
$1,00,000$ and $2,00,000,16 \%$ had their income between $2,00,000$ and $5,00,000$ and $14.3 \%$ had it above $5,00,000$ (Figure 3). This clearly shows the research was conducted among people belonging to adequately well established and settled backgrounds, still the lack of awareness should be considered as a reality check.

Figure 1. Gender distribution

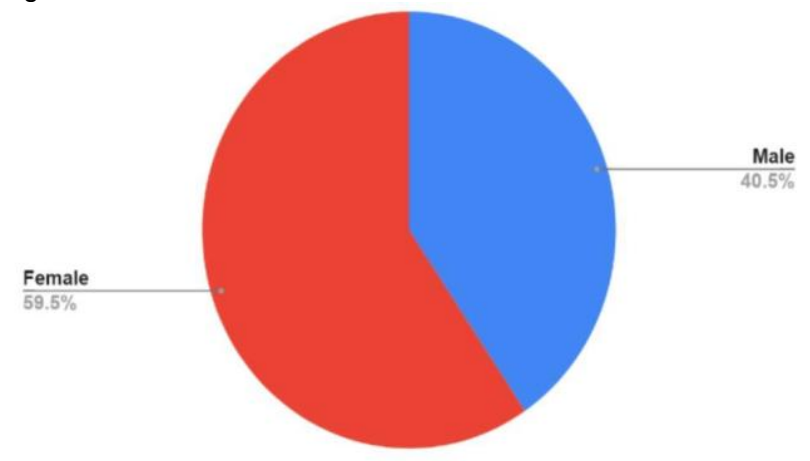

Figure 2.a. Are you a student? (i). If you are a student, are you a medical or a non-medical student? (ii). If you are not a student, please specify your employment status.

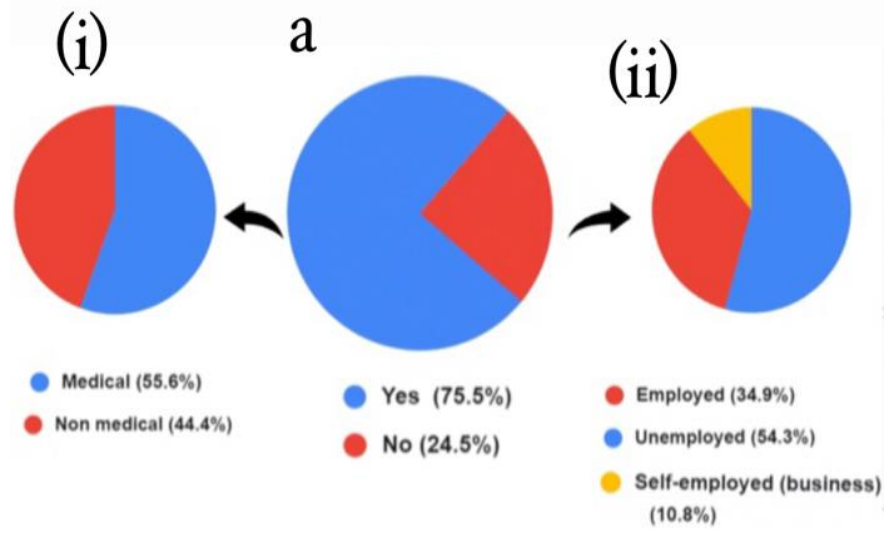

Figure 3. Distribution of the population under study based on monthly household income in PKR

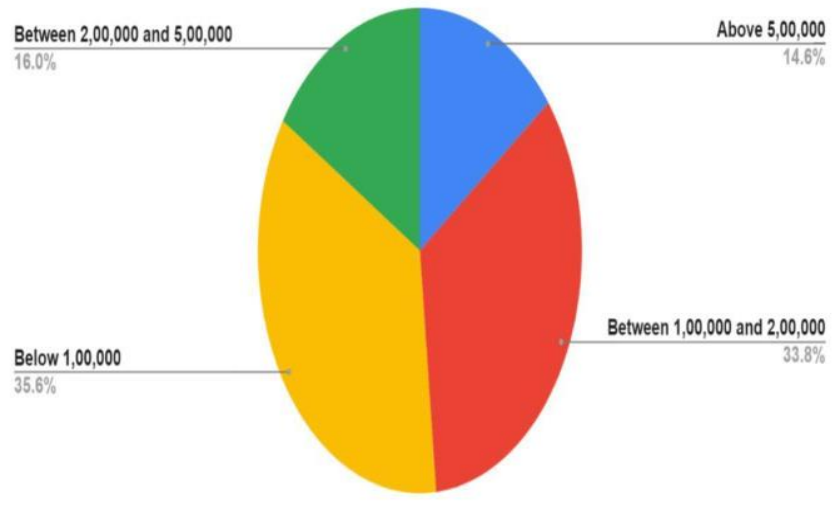


Figure $4^{*}$. To what extent do you agree with the statement that a woman with end-stage cancer, on multiple anticancer drugs and painkillers, should terminate her pregnancy?

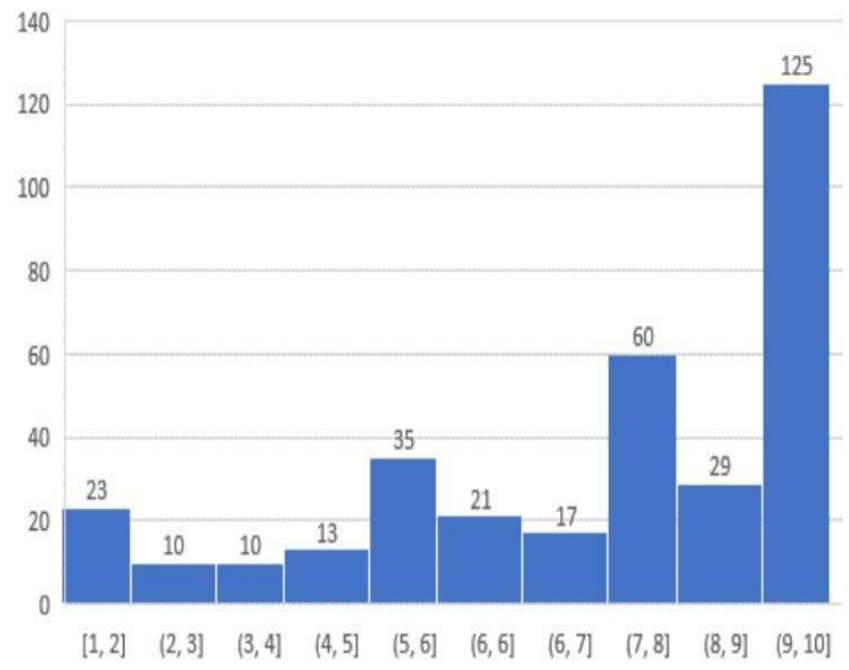

Figure $5^{\star}$. To what extent do you agree that it is okay for a woman to go through abortion if the pregnancy poses a serious life long threat to her health?

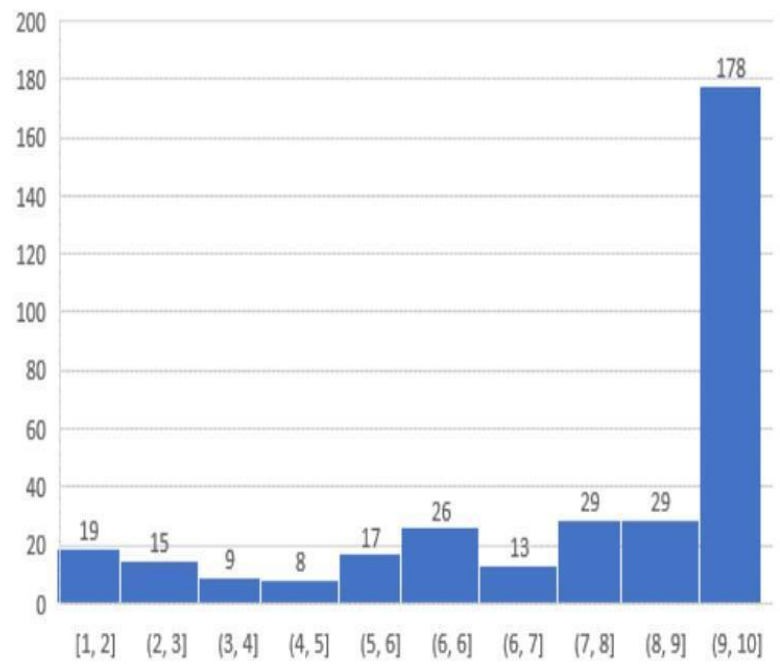

Figure $6^{*}$. In your opinion, should abortion be a choice for a depressed woman who has gone through a major trauma?

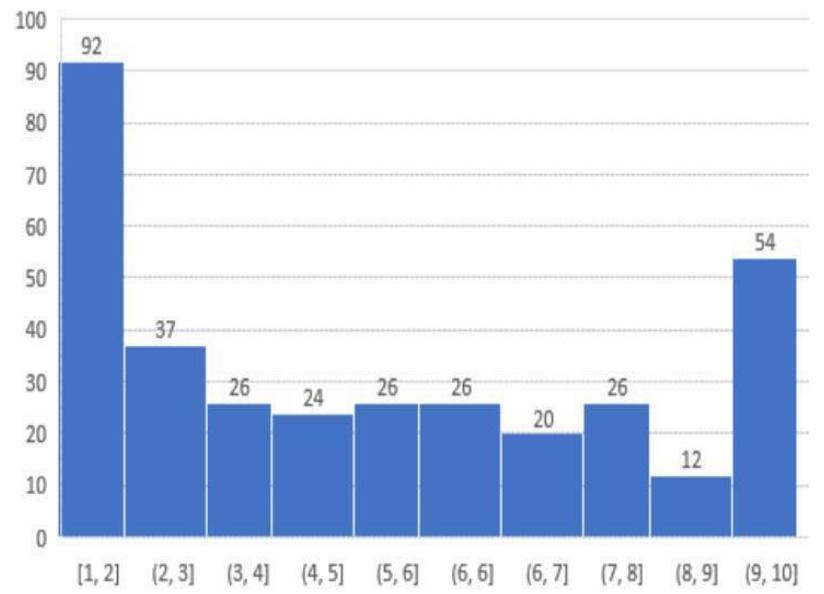

Figure $7 *$. To what extend do you agree that a basic level education regarding human development will contribute to the spread of awareness regarding abortion?

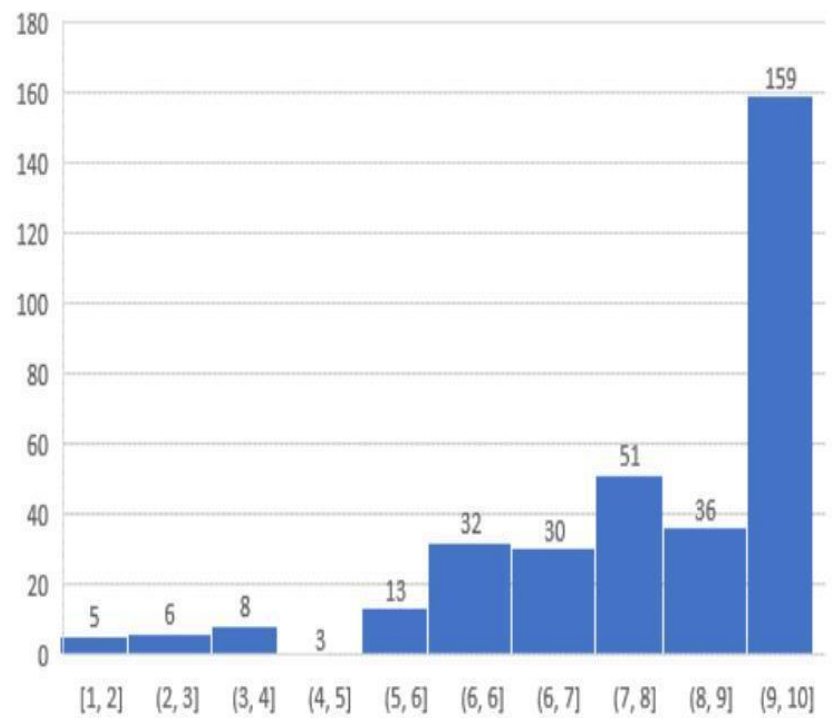

*The figures labelled so are the graphs obtained from the linearly-scaled, scenario-based questions with 1 being totally disagree and 10 being totally agree; the $x$ axis shows the linear scale and the $y$ axis shows the frequency of individuals opting for a number within the range of the linear scale corresponding to their opinion.

In the succeeding section, several scenario-based questions involving medical and social circumstances were given and the individuals were asked on whether they agree for a woman to opt for abortion, if she has to go through them during her pregnancy or not from a linear scale with 1 being totally disagree and 10 being totally agree. For better understanding and to authenticate the findings, we translated all the questions in the form circulated into Urdu as well.

In response to the question regarding the termination of pregnancy of a woman with end stage cancer, a total of $251(73 \%)$ tipped towards agreeing that abortion is a viable option in the given scenario while $57(16 \%)$ disagreed abortion under such circumstances and around 35(10\%) neither agreed nor disagreed with abortion as an option (Figure 4).

Similarly, in response to abortion being an option if it poses serious health afflictions to the mother herself, $309(90 \%)$ of the participants agreed to terminate the pregnancy while only about $34(9 \%)$ disagreed with the practice (Figure 5).

Contrastingly, majority of the participants disagreed with the statement that abortion should be allowed in cases of depression 179(52\%), however, a smaller majority $138(40 \%)$ held the opposite view that allowed depression as a valid reasoning for abortion (Figure 6).

Lastly, regarding the awareness towards abortion, a clear-cut majority $306(89 \%)$ was of the view that a basic root level education regarding human physiology will help the people to understand the practice of abortion and greatly de-stigmatize it (Figure 7). 


\section{DISCUSSION}

In order to gather the public's general perception regarding abortion and to what extend should it be legal, a programmed on-stream/web-based response form; comprising of scenario-based questions regarding the legitimacy of abortion under certain physical and mental health circumstances, was circulated among the student community of various undergraduate institutions and among adults of varying employment statuses.

As for the disease related scenarios, starting from cancer, the people were provided with the scenario of a woman with end stage cancer and on multiple chemotherapeutic drugs and painkillers, to choose whether she should terminate her pregnancy or not. ${ }^{10} \mathrm{~A}$ majority were of the view that chemotherapeutic drugs and painkillers are causes viable enough to opt for abortion. In conclusion, cancer among the general population is considered a disease serious enough and the general awareness of people towards cancer is adequate enough to deem the woman unfit to be a mother in case of unplanned pregnancy.

Induced abortions are medically encouraged witin the first trimester due to the inability of the mother to conceive the child. Moreover, it can lead to severe teratogenic effects in the foetus as well during as early as embryonic level. ${ }^{11}$

In order to draw out more inclusive results regarding the women's physical health we asked if it was viable to go through the entire pregnancy if it poses serious health issues for the mother herself. The outcome decisively concluded that almost all people are considering that the health of the woman is above everything and the pregnancy shouldn't be continued if it's a threat to the health of the woman. ${ }^{12}$ The clear consensus of people to allow abortion in such cases could be due to the mutual understanding that the law itself allows abortion in conditions like these as one of the comments in the online questionnaire said:

"I 99\% disagree with the concept [of abortion]. [it should be allowed] Only in one condition when mother's health is at stake."

Eleven other accounts in the questionnaire showed people's understanding that the mother's physical health is important enough to not be compromised.

Every year, millions of pregnant women are confronted with serious medical illnesses including hypertension, diabetes, autoimmune diseases such as arthritis and lupus, influenza, significant psychiatric illness, and even cancers. In short, gestation engenders a host of pregnancy-specific conditions that range from difficult (extreme nausea and vomiting) to disabling (sciatic nerve compression) to lifethreatening for the woman or her foetus (preeclampsia). ${ }^{13}$ Likewise, pulmonary hypertension carries a significant risk to not only the mother but the child alike during pregnancy. 14 Similarly, the medications that the women may take during their pregnancy could have severe teratogenic effects on the foetus itself. In addition to the teratogenic effects due to chemotherapeutics as explained above, there are other medicines including numerous anticoagulants (e.g. warfarin) that can lead to severe foetal anomalies. ${ }^{15}$
Contrary to the perception about physical health, the general attitude of the public towards depression and motherhood was somewhat careless with $51 \%$ of them disagreeing with regards to depression being an understandable reason behind abortion. The results could've been towards people agreeing to abort pregnancy if the woman's mental health is compromised as traumas can result in severe disabilities, physical and mental including depression and anxiety, ${ }^{16}$ which could drastically decrease the standards of living for the child itself. However, public's mutual disagreement highlights their perception that a new being, the bundle of joy, can erase past traumas as concluded by the people interviewed regarding abortion. Furthermore, a child can fill the void of the past traumas and lost souls and can prove to be the companion of one's golden age. Hence, interestingly enough people did not incline towards abortion as a considerable option for women with mental health issues as accounted by the people in the questionnaire:

"I don't know how people can kill their own child"

Another one said: "It feels so ruthless and shame[ful] that one [would] try to kill her own child, like the time of Jahiliya".

The objective of this paper was not just to understand the social trend regarding abortion but to excruciate a possible solution regarding the general awareness as well. Similar to work done by other scholars, including the likes of Tooley M. ${ }^{17}$ Hence, we asked the people to tell us whether a basic level education would help in achieving that or not, to which people wholly agreed.

Two points were proved: the first one being people's eagerness to have an understanding regarding abortion despite it being religiously and legally restricted and the second one being the mutual consensus of the general population that currently, people are inadequately educated regarding human embryology and physiology. One of the accounts had the following to say regarding abortion:

"In my opinion there should be proper educated seminars and campaigns to spread awareness among the masses especially to those who are illiterate. And not authentic facts regarding this sensitive matter should be regulated rather than people's opinions."

Another one of the comments added: "I think there is very less awareness about [the] topic of abortion. It is an important aspect of life and must be discussed by qualified panel of doctors and psychiatrist and religious scholars to discuss religious, medical and mental issues pertaining to abortion".

The last open- ended question was regarding people's general opinion on abortion in which mostly people supported the idea of abortion as long as the woman's physical health was endangered; however, the disregard towards it as a procedure practicable by women for their own mental health was evident.

\section{CONCLUSION}

The majority of the studied population was in support of abortion under the given circumstances. This was a result found by Jones R.K. and Jerman J. in their works done prior $^{18}$. Given our well designed and well-structured questionnaire, we were able to gauge opinions with great 
nuance and detail. Thus, we could infer that our population concurred that wherever, and whenever, external factors negatively impacted pregnancy, the pregnant individual should be allowed to terminate her pregnancy. This finding was particularly strongly correlated with the question, "to what extent do you agree with the statement that a woman with end-stage cancer, on multiple anti-cancer drugs and painkillers, should terminate her pregnancy?" Upwards of 120 agreed that, under these circumstances, a woman should terminate her pregnancy as the risk to the infant was too high. Similarly, positive results were found for questions about long-term health risks to the mother $(>80)$, as is the to be expected by past scholarly work ${ }^{19}$.

The discrepancy among the population under study begins to show when the questions begin to leave the confines of what is uncontrollable, to what is controllable. Contradictory to the above outcome, negative results were found for questions where the affliction for the mother and the child were less clear cut: with our question discussing depression and maternity, upwards of 90 people strongly disagreed with the mental state being a valid reason for abortion. This finding was hinted at by previous works as well, but we have confirmed these findings ${ }^{20}$.

However, regardless of the age and gender a clearcut majority agreed with abortion being an issue that is inadequately discussed and much awareness towards it is necessary especially from a biological perspective; one that is devoid of all social and religious taboos.

Acknowledgements: We express special gratitude to $\mathrm{CMH}$ Lahore Medical College and Institute of Dentistry for supporting our research and facilitating us in all regards possible.

Conflict of interest: The authors declare that there no conflicts of interest

\section{REFERENCES}

1. Marecek J, Macleod C, Hoggart L. Abortion in legal, social, and healthcare contexts. Feminism \& Psychology [Internet]. 2017 Feb;27(1):4-14. Available from: http://dx.doi.org/10.1177/0959353516689521

2. Kirkman M, Apicella C, Graham J, Hickey M, Hopper JL, Keogh L, et al. Meanings of abortion in context: accounts of abortion in the lives of women diagnosed with breast cancer. BMC Women's Health [Internet]. 2017 Apr 5;17(1). Available from: http://dx.doi.org/10.1186/s12905-017-0383-1

3. Stanley $\mathrm{KH}$, Susheela $\mathrm{S}$ and Taylor $\mathrm{H}$. The Incidence of Abortion Worldwide. International Family Planning Perspectives. 2018;25: 30-38.

4. Guttmacher institute . Unintended Pregnancy and Induced Abortion In Pakistan. [Online]. Available from: https://www.guttmacher.org/fact-sheet/unintended-pregnancy- and-induced-abortion-pakistan [Accessed 10 November, 2020].

5. Lohr PA, Hayes JL, Gemzell-Danielsson K. Surgical versus medical methods for second trimester induced abortion. Cochrane Database of Systematic Reviews [Internet]. 2008 Jan 23; Available from: http://dx.doi.org/10.1002/14651858.CD006714.pub2

6. Jones RK, Darroch JE, Henshaw SK. Contraceptive Use among U.S. Women Having Abortions in 2000-2001. Perspectives on Sexual and Reproductive Health [Internet]. 2002 Nov;34(6):294. Available from: http://dx.doi.org/10.2307/3097748

7. Stephanie W. Abortion options by trimester, healthline. [Online]. Available from: https://www.healthline.com/health/types-of-abortion [Accessed 10 November 2020].

8. Butler, L. K. (2015). Complexity of People's Attitudes Towards Abortion. Health, Human Performance and Recreation Undergraduate Honors Theses Retrieved from https://scholarworks.uark.edu/hhpruht/5

9. Agadjanian V, 2002. Is 'Abortion Culture' Fading in the Former Soviet Union? Views about Abortion and Contraception in Kazakhstan. Studies in Family Planning; 33(3): 237-248.

10. Thomson JJ. A defense of abortion. In Biomedical ethics and the law 1976 (pp. 39-54). Springer, Boston, MA.

11. J Arnon, D Meirow, H Lewis-Roness, A Ornoy. Genetic and teratogenic effects of cancer treatments on gametes and embryos. Human Reproduction Update. 2001;7(4): 394-403.

12. Kumar A, Hessini L, Mitchell EM. Conceptualising abortion stigma. Culture, health \& sexuality. 2009 Aug 1;11(6):625-39.

13. Margaret OL, Anne DL and Ruth RF. Pregnant women \& medical research: a moral imperative. Bioethica Forum. 2009;2(2): 60-65

14. BPmadden. Pulmonary hypertension and pregnancy. International Journal of Obstetric Anesthesia . April 2009;18(2): 156-164

15. Sanjay Raghav, David Reutens. Neurological sequelae of intrauterine warfarin exposure. Journal of clinical neuroscience. 2007;14(2): 99-103

16. Khan QU. Relationship of Salivary Cortisol Level With Severe Depression and Family History. Cureus [Internet]. 2020 Nov 18

17. Tooley M. Abortion and infanticide. New York. 1983.

18. Jones RK, Jerman J. Population group abortion rates and lifetime incidence of abortion: United States, 2008-2014. American journal of public health. 2017 Dec;107(12):1904-9.

19. Singh S, Shekhar C, Acharya R, Moore AM, Stillman M, Pradhan MR, Frost JJ, Sahoo H, Alagarajan M, Hussain R, Sundaram A. The incidence of abortion and unintended pregnancy in India, 2015. The Lancet Global Health. 2018 Jan 1;6(1):e111-20.

20. Puri M, Singh S, Sundaram A, Hussain R, Tamang A, Crowell $M$. Abortion incidence and unintended pregnancy in Nepal. International perspectives on sexual and reproductive health. 2016 Dec 1;42(4):197. 